\title{
Optimizing Wireless Access Networks towards Power Consumption: Influence of the Optimization Algorithm
}

\author{
Margot Deruyck, David Plets, \\ Luc Martens, Wout Joseph \\ Department of Information Technology \\ Ghent University/iMinds - WAVES \\ Ghent, Belgium \\ margot.deruyck@intec.ugent.be
}

\author{
Sotirios K. Goudos \\ Department of Physics \\ Aristotle University of Thessaloniki \\ Thessaloniki, Greece \\ sgoudo@physics.auth.gr
}

\begin{abstract}
Nowadays, wireless access networks are already amongst the top power consumers in the ICT (Information and Communication Technology) sector. As it expected that these networks will further expand in the future due to the extreme growth in mobile devices and the high bit rate demand of the applications running on these devices, it is important to consider power consumption as a key parameter in the network design phase. In this paper, two optimization algorithms are proposed: a capacity-based heuristic which aims to reduce power consumption by responding to the instantaneous bit rate demand by the user and an evolutionary opposition-based learning algorithm focusing on the joint-optimization of power consumption and geometrical coverage. Applying both algorithms on a realistic suburban case in Ghent, Belgium, show that both algorithms are able to design an LTE-A network consuming only $24 \%$ and $29 \%$, respectively, of the power consumed by the reference scenario which is representative for today's networks. The evolutionary algorithm outperforms the capacity-based algorithm by obtaining a $5 \%$ lower power consumption, while the capacity-based heuristic has a 2 to $3 \%$ higher coverage. Future research in joint-optimization algorithms of energy and network performance is definitely needed.
\end{abstract}

Keywords-energy efficiency; LTE-A; network design; optimization algorithm; power consumption; wireless access networks

\section{INTRODUCTION}

In the future, power consumption will become a key parameter when developing wireless access networks. From 2007 till 2012, the power consumption of today's wireless access networks has yearly increased with $10 \%$ [1]. If we look at the expectations for the near future [2], these wireless access networks will only need to expand in order not only to serve the extreme growth of mobile devices but also to support the higher bit rate required by the applications running on these devices. As the base station is the large power consumer in the wireless access network, in literature, a lot of attention has been given to determine and improve its power consumption in different circumstances. However, on network level, the work that has been done is limited [4], [5], [6], [7].
In this paper, two algorithms are proposed which aim to design the wireless network optimized towards power consumption, while preserving QoS (Quality of Service). The first one is a capacity-based heuristic, meaning that it will respond to the instantaneous bit rate demand of the user in order to develop an energy-efficient network. The second one is an evolutionary opposition-based learning algorithm focusing on the jointoptimization of the power consumption and the geometrical coverage. Both algorithms are applied on a realistic suburban area in Ghent, Belgium for two cases. Based on the obtained results, a comparison is made between both the energy and the network performance of both algorithms for a $4 \mathrm{G}\left(4^{\text {th }}\right.$ Generation) LTE-A (LTE Advanced) network.

This paper is organized as follows. In Section II, both algorithms are discussed in detail and the considered scenario is proposed. Section III compares the performance of both algorithms for the suburban scenario. In Section IV, our final conclusion is given.

\section{METHODOLOGY}

\section{A. Capacity-based heuristic}

As mentioned above, the first algorithm is a capacity-based heuristic which will respond to the instantaneous bit rate demand of the users in the considered area.

1) Input:

Before we can actually start designing the network, some input is required:

- The considered area: the area is identified by a shape file, describing the different buildings (location, height, etc.) in the environment.

- A list of possible base locations: this list consists of all the existing base station locations in the considered area.

- A list of users with their required bit rate: this list tells us the location of all the users active in the considered area together with the bit rate they require. The number of users depends on the population density of the considered area and is obtained from processing measurements [3]. The worst case scenario i.e., the 
time during the day where there are most users active is considered (around 5 p.m.). The users are uniform distributed over the considered area i.e., each location in the area has the same chance to be chosen as user location. For the bit rate distribution, two bit rates are considered: $64 \mathrm{kbps}$ (voice call) and $1 \mathrm{Mbps}$ (data call). The amount of users making a voice call or a data call is based on confidential data from an operator.

\section{2) Algorithm:}

Fig. 1 shows the flow diagram for the different steps of the algorithm. Based on the list of users we need to cover, a first network consisting only of femtocell base station is developed. It will of course not be possible to cover all users by femtocell base stations only. Based on the list of users we were not able to cover by femtocell base stations only, a second network is developed by using macrocell base stations as shown in Fig. 1. the algorithm.

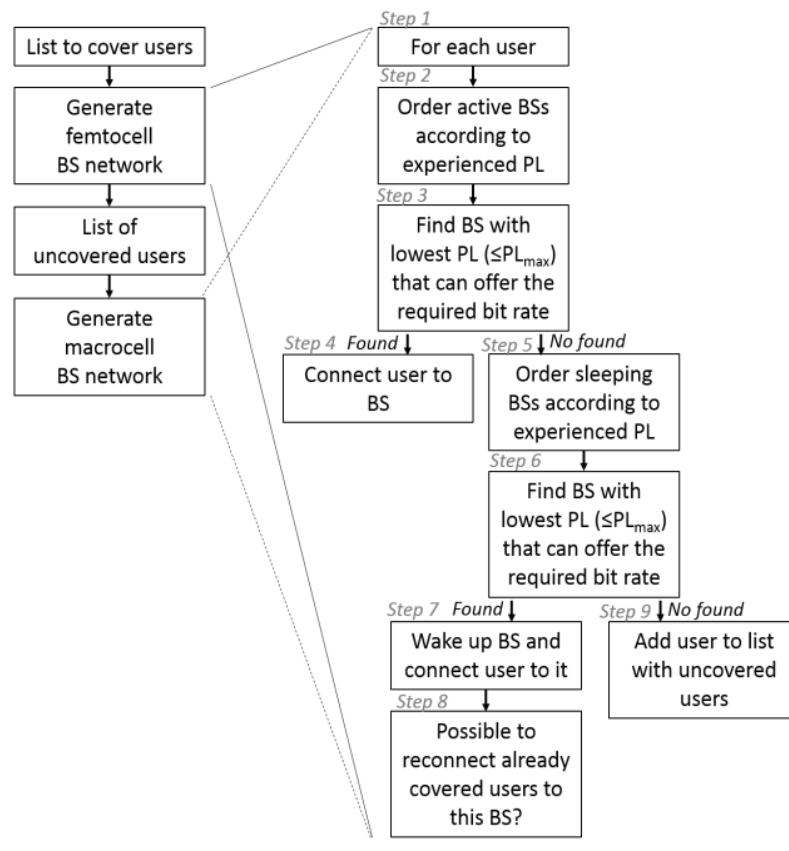

Figure 1 Flow diagram of the algorithm.

To generate the femtocell and macrocell network, the same approach is used (Fig. 1 Step 1 to 9). For each user on the 'to cover' list (Fig. 1 Step 1), we try to find a base station (BS) the user could connect to. As it is more energy-efficient to connect a user to an already active base station, instead of waking one up, we first try to find an already active base station (Fig. 1 Step 2 ). To this end, the active base stations are ordered according to the path loss (PL) experienced by the user. Next, we go over this list and look for the base station from which the user experiences the lowest path loss and can still offer the bit rate required by the user. The experienced path loss should of course be lower than the maximum allowable path loss $\left(P L_{\max }\right)$ to which a transmitted signal can be subjected while still having a sufficient quality at the receiver side. One of the key parameters to determine $P L_{\max }$ is the receiver SNR (Signal-to-Noise Ratio) which describes the sensitivity of the receiver and depends on the bit rate required by the user as discussed in [8]. If such a base station can be found, the user is connected to it (Fig. 1 Step 4) and the algorithm can continue with the next user. Otherwise, the same procedure is repeated for all the sleeping base stations (Fig. 1 Step $5 \& 6$ ). In case a sleeping base station that match the criteria is found, it is turned on and the user is connect to it (Fig. 1 Step 7). Furthermore if a sleep base station is switched on, the algorithm checks if it is possible to reconnect already covered users to this base station in case they experience a lower path loss from this 'new' base station (Fig. 1 Step 8). This step is needed in order to balance the load over all active base stations in the network. In case it is not possible to cover a user by an active nor by a sleeping base station, it will not be able to cover the user and the user is added to the uncovered users list (Fig. 1 Step 9).

\section{B. Modified Oppositional Biogeography-Based Optimization $(\mathrm{MOBBO})$}

The second algorithm we consider is an evolutionary algorithm based on mathematical models that describe how species migrate form one island to another, how new species arise, and how species become extinct. The version considered here is based on semi-opposite points as proposed in [12]. BBO solutions share directly their attributes using the migration models. The migration operator provides BBO with a good exploitation ability. Due to these differences, BBO can outperform other algorithms [9], [10], [11]. Note that if other algorithms like PSO (Particle Swarm Optimization) and DE (Differential Evolution) are constrained to discrete space then the next generation will not necessarily be discrete [11]. However, this is not true for BBO. If BBO is constrained to a discrete space then the next generation will also be discrete. As suggested in [11], this indicates that BBO could perform better than other EAs on combinatorial optimization problems, which makes BBO suitable for application to energy-efficient network design.

The following parameters are used:

- A control parameter named opposition probability $p_{0}$ $(\in[0,1])$ : this parameter controls if a SIV (Suitable Index Variable) variable in a habitat will be replaced by its opposite or not.

- A jumping rate parameter $j_{r}(\in[0,1])$ : this parameter controls in each generation if the opposite population is created or not.

Furthermore, two additional parts compared to the original algorithm code are required. The first one is the oppositionbased population initialization which is described below (Algorithm 1) [12]. low $w_{j}$ and upper $_{j}$ are the lower and the upper limit in the $\mathrm{j}$-th dimension respectively.

The second additional part is the opposition-based generation jumping. A description of the algorithm in pseudo-code is given below (Algorithm 2) [12]. The $\min _{j}$ and $\max _{j}$ are the minimum and maximum values of the $\mathrm{j}$-th dimension in the current population respectively. 

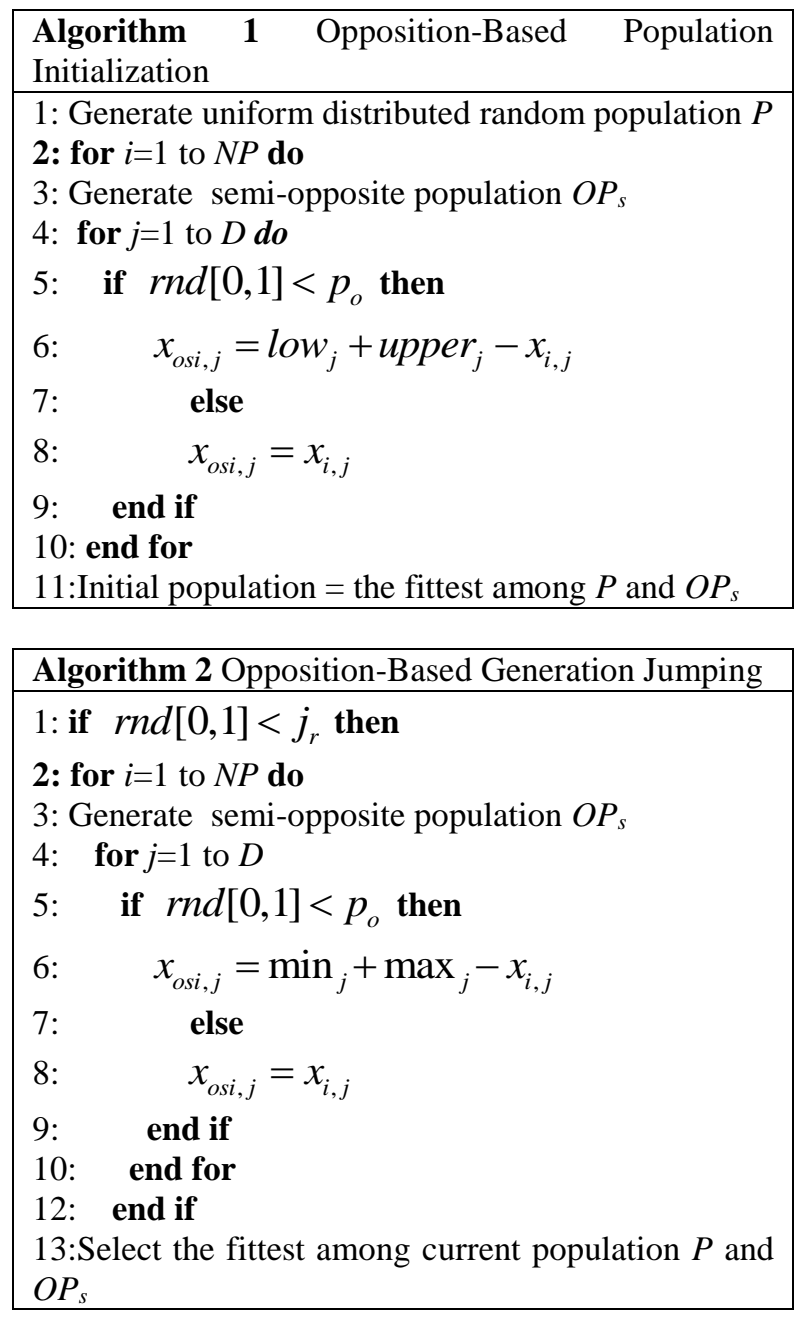

The MOBBO code algorithm can then be described as follows [12]:

1. Initialize the MOBBO control parameters $p_{o}$ and $j_{r}$.

2. Initialize a random population of $N P$ habitats (phase vectors) from a uniform distribution.

3. Set the number of generations $G$ to one.

4. Initialize the opposite population according to Algorithm 1.

5. Map the $H S I$ value to the number of species $\mathrm{S}$, the immigration rate $\lambda_{k}$, the emigration rate $\mu_{k}$ for each solution (phase vector) of the population.

6. Apply the migration operator for each non-elite habitat based on immigration and emigration rates using the following formulas

$\mu_{k}=E\left(\frac{k}{S_{\max }}\right), \quad \lambda_{k}=I\left(1-\frac{k}{S_{\max }}\right)$

[12]:

with $I$ the maximum possible immigration rate, $E$ the maximum possible emigration rate, $k$ is the rank of the given candidate solution, and $S_{\max }$ the maximum number of species (e.g., population size). The rank of the given candidate solution or the number of species is obtained by sorting the solutions from most fit to least fit according to the HSI value.
7. Apply the mutation operator.

8. Evaluate objective function value [12]:

$H S I=F(\tilde{x})=-\left(f_{\text {cov }}(\tilde{x})+k \cdot f_{\text {pow }}(\tilde{x})\right)$ with

$$
\begin{gathered}
f_{\text {cov }}(\tilde{x})=100 \cdot \frac{A_{\text {target }} \cap A(\tilde{x})}{A_{\text {target }}} \\
f_{\text {pow }}(\tilde{x})=100 \cdot\left(1-\frac{P(\tilde{x})}{P_{\max }}\right)
\end{gathered}
$$

$$
k=\left\{\begin{aligned}
0, & f_{\text {cov }}(\tilde{x})<90 \\
\frac{\left(f_{\operatorname{cov}}(\tilde{x})-90\right)^{2}}{5}, & 90 \leq f_{\text {cov }}(\tilde{x})<95 \\
5, & \text { else }
\end{aligned}\right.
$$

9. If $r n d[0 ; 1]<j_{r}$ calculate the opposite population according to Algorithm 2.

10. Repeat Step 5 until the maximum number of generations $G_{\max }$ or the maximum number of objective function evaluations is reached.

\section{Scenario}

For this study, the target area shown in Fig. 2 is considered. This is an outdoor suburban area of $6.85 \mathrm{~km} 2$ in the city center of Ghent, Belgium. The 75 possible locations for the base station are indicated by red squares in Fig. 2. These are existing base station locations, located on the roofs of buildings. In the considered area, 224 users are active (worst case scenario [14]), requiring $64 \mathrm{kbps}$ (voice call) or 1 Mbps (data call) according to the distribution proposed in [14].

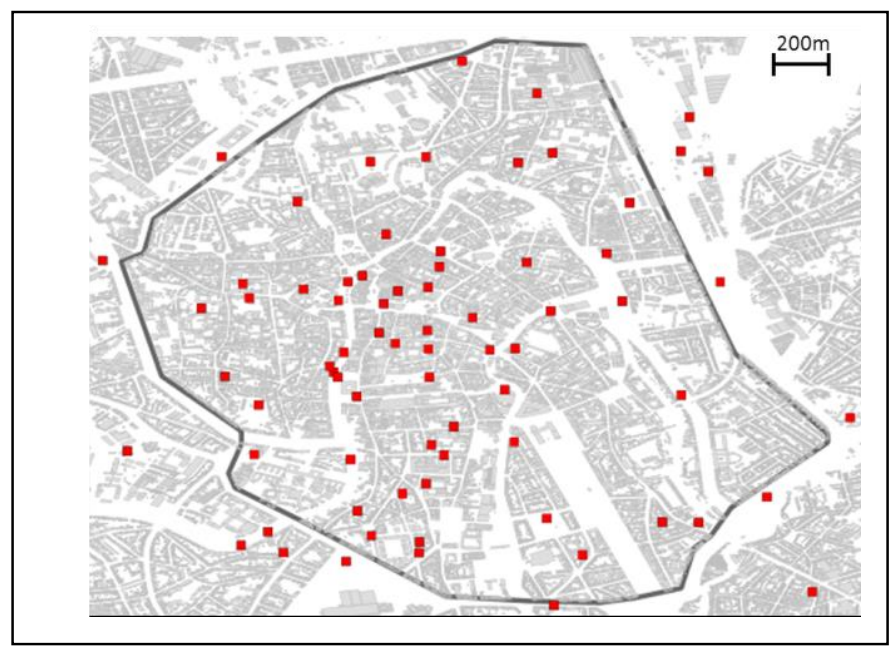

Figure 2 The considered suburban area of $6.85 \mathrm{~km}^{2}$ in Ghent, Belgium. The red squares represents the possible base station locations.

Furthermore, LTE-A is used as wireless technology. The assumed link budget parameters for both the macrocell and the femtocell base station are summarized in Table I.

To calculate the power consumption of the network, the models for the power consumption of the macrocell and femtocell base station of [13] are used. As reference scenario, we assume that all 75 base stations active and are considered as macrocell base stations, operating on their highest power consumption i.e., $1.7 \mathrm{~kW}[13]$. 
Finally, the algorithms are compared for two case. In the first case, the network consist only of macrocell base stations, while in the second case, the network consists of a mixture of macrocell and femtocell base stations.

Table 1 Link budget parameters for the LTE-A macrocell and femtocell base

\begin{tabular}{|l|l|l|}
\hline \multicolumn{1}{|c|}{ Parameter } & \multicolumn{1}{|c|}{ Macrocell BS } & \multicolumn{1}{c|}{ Femtocell BS } \\
\hline Frequency & $2.6 \mathrm{GHz}$ & $2.6 \mathrm{GHz}$ \\
\hline $\begin{array}{l}\text { Maximum input power } \\
\text { base station antenna }\end{array}$ & $43 \mathrm{dBm}$ & $33 \mathrm{dBm}$ \\
\hline $\begin{array}{l}\text { Antenna gain of base } \\
\text { station }\end{array}$ & $18 \mathrm{dBi}$ & $4 \mathrm{dBi}$ \\
\hline Antenna gain of receiver & $0 \mathrm{dBi}$ & $0 \mathrm{dBi}$ \\
\hline Feeder loss base station & $2 \mathrm{~dB}$ & $2 \mathrm{~dB}$ \\
\hline Feeder loss receiver & $0 \mathrm{~dB}$ & $0 \mathrm{~dB}$ \\
\hline Fade margin & $10 \mathrm{~dB}$ & $10 \mathrm{~dB}$ \\
\hline Interference margin & $2 \mathrm{~dB}$ & $2 \mathrm{~dB}$ \\
\hline Noise figure of receiver & $8 \mathrm{~dB}$ & $8 \mathrm{~dB}$ \\
\hline $\begin{array}{l}\text { Implementation loss of } \\
\text { receiver }\end{array}$ & $0 \mathrm{~dB}$ & $0 \mathrm{~dB}$ \\
\hline MIMO & $1 \times 1$ & $1 \mathrm{x} 1$ \\
\hline Receiver SNR & $1 / 3 \mathrm{QPSK}=-1.5 \mathrm{~dB}$ \\
& $1 / 2 \mathrm{QPSK}=3 \mathrm{~dB}$ & \\
& $2 / 3 \mathrm{QPSK}=10.5 \mathrm{~dB}$ & \\
& $1 / 216-\mathrm{QAM}=14 \mathrm{~dB}$ & \\
& $2 / 316-\mathrm{QAM}=19 \mathrm{~dB}$ \\
& $1 / 264-\mathrm{QAM}=23 \mathrm{~dB}$ \\
& $2 / 364-\mathrm{QAM}=29.4 \mathrm{~dB}$ \\
\hline Bandwidth & $5 \mathrm{MHz}$ & $5 \mathrm{MHz}$ \\
\hline $\begin{array}{l}\text { Soft handover gain } \\
\text { receiver }\end{array}$ & $0 \mathrm{~dB}$ & $0 \mathrm{~dB}$ \\
\hline Building penetration loss & $0 \mathrm{~dB}$ (only outdoor coverage considered) \\
\hline Height mobile station & $1.5 \mathrm{~m}$ & $1.5 \mathrm{~m}$ \\
\hline
\end{tabular}

\section{COMPARISON OF THE ALGORITHMS}

In this section, the performance of both algorithms is compared. The MOBBO algorithm is executed 20 times. The population size is set to 100 and the maximum number of generations to 1000 iterations. Furthermore, the number of objective function evaluations is limited to 100000 . For the capacity-based algorithm, the algorithm is executed 40 times, due to the variation of the user location and user bit rate distribution. The mean value is considered over all simulations. Table II shows the results obtained with both algorithms. The reference scenario has a power consumption of $127.5 \mathrm{~kW}$ with a geometrical coverage of $100 \%$. To this end, 75 base stations are used. Note that the reference scenario for both cases (the macrocell only network and the macrocell femtocell network) is the same.

Table 2 Comparison of the capacity-based and the MOBBO algorithm for the considered cases.

\begin{tabular}{|l|l|l|l|l|}
\hline Case & Algorithm & Macro/Femto & $\begin{array}{l}\text { Power } \\
\text { consumption }\end{array}$ & $\begin{array}{l}\text { Geometrical } \\
\text { Coverage }\end{array}$ \\
\hline Macro & Reference & 75 & $100 \%$ & $100 \%$ \\
Only & MOBBO & $20 / 0$ & $24.5 \%$ & $95 \%$ \\
& Capacity & $29 / 0$ & $30.2 \%$ & $98.4 \%$ \\
\hline Macro & MOBBO & $20 / 3$ & $24.4 \%$ & $95 \%$ \\
+femto & Capacity & $28 / 29$ & $29.3 \%$ & $97.1 \%$ \\
\hline
\end{tabular}

For the macrocell only case the MOBBO algorithm uses 20 macrocell base stations, resulting in a power consumption of $24.5 \%$ compared to the reference scenario. A geometrical coverage of $95 \%$ is obtained. The MOBBO performs better than the capacity based algorithm which uses 29 macrocell base stations resulting in a power consumption of $30.2 \%$ compared to the reference scenario, but the geometrical coverage is also higher than for the MOBBO case (98.4\% versus $95 \%)$.

Both algorithms have a slightly lower power consumption when introducing femtocell base stations. For the MOBBO algorithm, a power consumption of $24.4 \%$ is obtained compared to $24.5 \%$ when only macrocell base stations are used. This very small difference is due to the fact that it only uses 3 femtocell base stations, while still using 20 macrocell base stations. Although the same number of macrocell base stations are used, the power consumption is slightly lower as it is possible to reduce the antenna's input power of some of the macrocell base stations due to the introduction of the femtocell base stations. The coverage is for both cases the same i.e., $95 \%$. For the capacitybased algorithm, the reduction in power consumption is higher, from $30.2 \%$ when using only macrocell base stations to $29.3 \%$ when using both femtocell and macrocell base stations. The power consumed by this network is still higher than the network obtained with the MOBBO algorithm as much more base stations are used: 28 femtocell base stations and 29 macrocell base stations. The fact that this algorithm uses much more femtocell base stations is due to the fact that we first try to cover as many users as possible by femtocell base stations. A decrease in geometrical coverage is also noticed: from $98.4 \%$ to $97.1 \%$.

Note that the power consumption reduction by using femtocell base stations is very limited in the considered case. This is probably due to the fact that femtocell base stations can only be placed on the existing locations of macrocell base stations. Future work will consist of allow other locations as well for the femtocell base stations.

In general, we can conclude that the MOBBO algorithm performs around $5 \%$ better in terms of power consumption, however, the capacity-based algorithm performs better in terms of geometrical coverage (around 2 to $3 \%$ higher).

\section{CONCLUSION}

Power consumption and energy efficiency are becoming more and more important in all aspects of our daily life. As wireless access networks are amongst the top power consumers in ICT (Information and Communication Technology), it will be necessary to consider the network's power consumption as well during the network design phase. Especially for the future where today's wireless access networks will need to expand in order to cope with the extreme growth of mobile devices and the high bit rate demand of the applications running on those devices. In this paper, two algorithms are proposed and compared to optimize the wireless network towards power consumption. The first algorithm is a capacity-based heuristic which tries to save energy by responding to the instantaneous bit rate demand of the user. The second algorithm is an evolutionary opposition-based 
learning algorithm focusing on the joint-optimization of power consumption and geometrical coverage. Both algorithms are applied on a realistic suburban area in Ghent, Belgium. Two LTE-A cases are considered. In the first case, a network consisting only of macrocell base stations is developed. In the second case, a mixture of macrocell and femtocell base stations is considered. Both algorithms accomplish to use only $24 \%$ and $29 \%$ of the power consumed by the reference scenario, where all base stations are active i.e., the situation nowadays. Comparing the algorithms for both cases shows that the evolutionary algorithm performs around 5\% better in terms of power consumption, while the network designed by the capacity-based algorithm has a 2 to $3 \%$ higher coverage. Depending on which parameter, power consumption or coverage, is the most important one, a different algorithm needs to be considered as shown by our results.

These preliminary results show that is interesting to consider and compare different optimization algorithms depending on which parameter is the key one. Further research will include to compare more optimization algorithms for multiple scenarios.

\section{ACKNOWLEDGMENT}

Margot Deruyck is supported by a postdoctoral grant from the Special Research Fund (BOF) of Ghent University (Belgium).

\section{REFERENCES}

[1] W. Van Heddeghem, S. Lambert, B. Lannoo, D. Colle, M. Pickavet, P. Demeester, "Trends in worldwide ICT electricity consumption from 2007 to 2012”, Computer Communications, Vol. 50, pp. 64-76, 2014.

[2] Cisco, "Visual Networking Index: Global Mobile Data Traffic Forecast 2015-2020", 2015.

[3] M. Deruyck, E. Tanghe, W. Joseph, L. Martens, "Characterization and optimization of the power consumption in wireless access networks by taking daily traffic variations into account", EURASIP Journal on Wireless Communication Networking, Vol. 248, 2012.

[4] M. Deruyck, W. Vereecken, W. Joseph, B. Lannoo, L. Martens, "Reducing the power consumption in wireless access networks: Overview and recommendations", Progress in Electromagnetics Research, Vol. 132, pp. 255-274, 2012.

[5] M.A. Marsan, L. Chiaraviglio, D. Ciullo, M. Meo, "A simple analytical model for the energy-efficient activation of access points in dense WLANs", Proceedings of the 1st International Conference on EnergyEfficient Computing and Networking (e-Energy), pp. 159-168, 2010.

[6] G. Koutitas, "Green network planning of single frequency networks", IEEE Transactions on Broadcasting, Vol. 56, No. 4, pp. 541-550, 2010.

[7] M. Deruyck, E. Tanghe, D. Plets, L. Martens, W. Joseph, “Optimizing LTE Wireless Access Networks towards Power Consumption and Electromagnetic Exposure of Human Beings", Elsevier Computer Networks, Vol. 94, pp. 29-40, 2016.

[8] M. Deruyck,W. Joseph, L. Martens, "Power consumption model for macrocell and microcell base stations", European Transactions on Emerging Telecommunications Technologies, Vol. 25, No. 3, pp. 320333, 2012.

[9] D. Simon, "Biogeography-Based Optimization", IEEE Transactions on Evolutionary Computation, Vol. 12, pp. 702-713, 2008.

[10] H. Ma, "An analysis of the equilibrum of migration models for biogeography-based optimization", Information Sciences, Vol. 180, pp. 3444-3464, 2010.

[11] H. Ma, D. Simon, M. Fei, Z. Chen, "On the equivalences and differences of evolutionary algorithms", Engineering Applications of Artificial Intelligence, Vol. 26, pp. 2397-2407, 2013.

[12] S.K. Goudos, M. Deruyck, D. Plets, L. Martens, W. Joseph, “Application of Opposition-Based Learning Concepts in Reducing the Power Consumption in Wireless Access Networks", 23rd International Conference on Telecommunications (ICT), 2016.

[13] M. Deruyck, W. Joseph, B. Lannoo, D. Colle, L. Martens, "Designing Energy-Efficient Wireless Access Networks: LTE and LTE-Advanced", IEEE Internet Computing, Vol. 17, No. 5, pp. 39-45, 2013.

[14] M. Deruyck, W. Joseph, E. Tanghe, L. Martens, "Reducing the power consumption in LTE-Advanced wireless access networks by a capacity based deployment tool”, Radio Science, Vol. 49, pp. 777-787, 2014. 